\title{
Incidência e mortalidade da sífilis congênita: Um estudo de série temporal
}

\author{
Incidence and Mortality of Congenital Syphilis: A time series study \\ Incidencia y mortalidad de la sífilis congènita: Un studio de series de tiempo
}

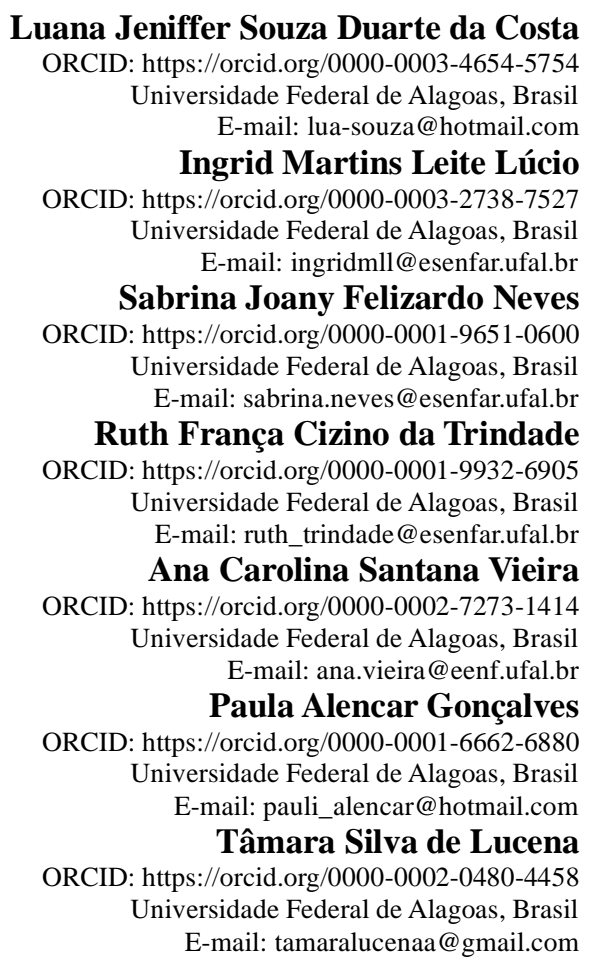

\begin{abstract}
Resumo
Objetivo: Descrever incidência e mortalidade da sífilis congênita no período de 2009 a 2018. Metodologia: Estudo epidemiológico, descritivo, com delineamento transversal. População composta por 1876 casos notificados de sífilis congênita em residentes de Maceió. Os dados foram extraídos dos Sistemas de Informações de Saúde. Para análise estatística utilizou-se os teste qui-quadrado, ANOVA e regressão Linear. A pesquisa foi aprovada sob parecer $\mathrm{n}^{\circ}$ 3.138.921. Resultados: A incidência de sífilis congênita apresentou tendência de aumento nos últimos 10 anos. Foram registrados 32 óbitos, o que correspondeu a letalidade de 1,7 óbitos/100 casos de sífilis congênita. A mortalidade infantil apresentou maior taxa em 2012, 0,47 óbitos/1000 nascidos vivos, mantendo um padrão regular entre os anos avaliados. Conclusão: A alta incidência de sífilis congênita em Maceió representa um desafio para a saúde pública e requer estratégias de melhoria no atendimento de pré-natal, afim de diminuir as taxas de mortalidade por esta causa.
\end{abstract}

Palavras-chave: Sífilis; Sífilis congênita; Incidência; Mortalidade; Estudos de séries temporais.

\begin{abstract}
Objective: To describe the incidence and mortality of congenital syphilis in the period from 2009 to 2018. Methodology: Epidemiological, descriptive study, with a cross-sectional design. Population composed of 1876 notified cases of congenital syphilis in Maceió residents. The data were extracted from the Health Information Systems. For statistical analysis, the chi-square test, ANOVA and Linear regression were used. The survey was approved under opinion $\mathrm{n}^{\mathrm{o}} 3.138 .921$. Results: The incidence of congenital syphilis has shown an increasing trend in the last 10 years. 32 deaths were recorded, which corresponded to a lethality of 1.7 deaths / 100 cases of congenital syphilis. Infant mortality showed the highest rate in 2012, 0.47 deaths / 1000 live births, maintaining a regular pattern between the years evaluated. Conclusion: The high incidence of congenital syphilis in Maceió represents a challenge for public health and requires strategies to improve prenatal care, in order to decrease mortality rates due to this cause. Keywords: Syphilis; Syphilis congenital; Incidence; Mortality; Time series studies.
\end{abstract}




\begin{abstract}
Resumen
Objetivo: Describir la incidencia y mortalidad de la sífilis congénita en el período de 2009 a 2018. Metología: Estudio epidemiológico, descriptivo, con diseño transversal. Población compuesta por 1876 casos notificados de sífilis congénita en residentes de Maceió. Los datos fueron extraídos de los Sistemas de Información en Salud, para el análisis estadístico se utilizó la prueba de chi-cuadrado, ANOVA y regresión lineal. La encuesta fue aprobada con el dictamen $\mathrm{n}^{\mathrm{o}}$ 3.138.921. Resultados: La incidencia de sífilis congénita ha mostrado una tendencia creciente en los últimos 10 años. Se registraron 32 muertes, lo que correspondió a una letalidad de 1,7 muertes / 100 casos de sífilis congénita. La mortalidad infantil mostró la tasa más alta en 2012, 0,47 muertes / 1000 nacidos vivos, manteniendo un patrón regular entre los años evaluados. Conclusión: La alta incidencia de sífilis congénita en Maceió representa un desafío para la salud pública y requiere estrategias para mejorar la atención prenatal, con el fin de disminuir las tasas de mortalidad por esta causa.
\end{abstract}

Palabras clave: Sífilis; Sífilis congénita; Incidencia; Mortalidad; Estudios de series temporales.

\title{
1. Introdução
}

A sífilis é uma doença crônica, infecciosa e sistêmica, conhecida desde o século XV, que afeta exclusivamente os seres humanos. É uma infecção bacteriana que tem como agente uma bactéria Gram-negativa do grupo das espiroquetas, nomeada como Treponema pallidum - T. pallidum (Gonçalves Trevisan et al., 2018; Mahmud et al., 2019; Neves et al., 2019). Apresenta-se nas formas adquirida, congênita e em gestante (Lafetá et al., 2016; Brasil, 2017).

Sífilis Congênita - SC ocorre por meio da disseminação hematogênica do T. pallidum da mãe para o feto, em qualquer momento da gestação, independente do estágio clínico da doença, em gestante não tratada ou tratada de forma inadequada, e nestes casos, transmissão por via transplacentária ou durante o parto (Barbosa et al., 2017; Moreira et al., 2017; Arruda \& Ramos, 2020). Nesse contexto, o risco de transmissão da doença para o feto está relacionado com o estágio da infecção materna e do período gestacional em que o feto é exposto, sendo menor em fases iniciais da gestação (Brasil, 2018a).

Para fins da vigilância epidemiológica, a SC no Brasil passou a ser considerada uma doença de notificação compulsória por meio da Portaria do Ministério da Saúde nº 542 de 22 de dezembro de 1986 (Barbosa et al., 2017; Moreira et al., 2017; Guimarães et al., 2018). A realização da notificação é obrigatória para todo profissional de saúde e o seu descumprimento confere infração à legislação sanitária (Lafetá et al., 2016).

Mundialmente, a doença atinge um milhão de gestantes por ano, contribuindo com mais de 300 mil mortes fetais e neonatais e colocando em risco de morte prematura mais de 215 mil crianças (Brasil, 2018b; Coelho et al., 2018). No Brasil ainda persiste como grave problema de saúde pública por permanecer entre as doenças transmissíveis que mais acometem o período gravídico puerperal, com taxas de infecção por transmissão vertical variando entre $30 \%$ a $100 \%$ dos casos entre as fases primária, secundária, latente tardia e terciária (Barbosa et al., 2017; Almeida \& Pinto, 2018; Brasil, 2019a).

Os recém-nascidos expostos a essa doença no período gestacional e sem o tratamento adequado podem desenvolver complicações perinatais adversas incluindo: abortamento, óbito fetal ou perinatal, parto prematuro, baixo peso ao nascer, anomalias congênitas, sífilis ativa no RN e sequelas a longo prazo, como surdez, cegueira, hidrocefalia e comprometimento neurológico (Boni; Pagliari, 2016; Domingues; Leal, 2016; Feitosa, Rocha \& Costa, 2016; Cerqueira et al., 2017).

Tais complicações trazem custos significativos para os serviços de saúde, com valores superiores aos cuidados aplicados a uma criança sem a infecção, necessitando ainda de acompanhamento por período prolongado e profissionais especializados. Esses gastos poderiam ser reduzidos com investimentos na prevenção, detecção precoce e tratamento oportuno (Costa et al., 2013; Sonda et al., 2013).

$\mathrm{Na}$ inexistência de tratamento da mãe, a transmissão vertical da sífilis é alta, podendo obter valores aproximados a $100 \%$ nas formas recentes da doença. Contudo, um diagnóstico e tratamento adequados são altamente efetivos e reduzem a transmissão vertical em até 97\% dos casos (Domingues \& Leal, 2016; Ferreira, Barbosa \& Maleck, 2018).

Embora haja um conhecimento acerca dos benefícios oriundos do diagnóstico e tratamento da sífilis, alguns fatores 
podem influenciar o controle da doença, por exemplo: dificuldade no acesso ou acesso tardio ao pré-natal, ausência ou baixa cobertura dos programas de prevenção, ausência da solicitação dos exames sorológicos, baixa adesão ao tratamento da gestante e parceiro, falta de conhecimento dos profissionais de saúde quanto as graves consequências da doença e estigmatização e discriminação com relação as infecções sexualmente transmissíveis (França et al., 2015; Beck \& Souza, 2018; Motta et al., 2018).

Partindo da hipótese de que a SC tem aumentado crescentemente sua incidência nos últimos anos, considera-se então que a cidade de Maceió apresenta valores significativos da doença, em virtude da baixa cobertura de atenção primária no município. De acordo com o Departamento de Saúde da Família, no período de setembro de 2019, aproximadamente 43,56\% da população tem acesso aos serviços de atenção básica, e apenas 27,13\% são assistidos pela Estratégia Saúde da Família, no município de Maceió (Brasil, 2019b).

Dessa forma, este estudo foi desenvolvido a partir da seguinte questão norteadora: Qual o perfil epidemiológico da sífilis congênita no município de Maceió nos últimos dez anos?

Levando em consideração o impacto da SC na assistência em saúde pública, a dificuldade de controle dessa infecção, as fragilidades encontradas pela vigilância epidemiológica, a fragmentação do cuidado e o emprego das políticas de saúde instituídas no âmbito mundial e nacional, este estudo torna-se relevante pela necessidade de investigação dos casos notificados de doença no município de Maceió, tendo em vista a elevação das taxas de incidência da doença no Estado de Alagoas nos últimos anos, considerando que a transmissão vertical ocorre por falha na assistência de pré-natal durante o diagnóstico e/ou tratamento dos casos.

Nessa perspectiva, o estudo teve por objetivo descrever a incidência e a mortalidade da sífilis congênita no período de 2009 a 2018 no município de Maceió, Alagoas.

\section{Metodologia}

Trata-se de um estudo epidemiológico descritivo, com delineamento transversal, destinado a avaliar a tendência temporal da taxa de incidência e mortalidade da sífilis congênita, na cidade de Maceió, nos anos de 2009 a 2018.

O estudo teve como área de abrangência o município de Maceió. Cidade capital do Estado de Alagoas, localizada na Região Nordeste do país. Ocupa uma área de abrangência de 509,552 km2 e representa, aproximadamente, 29,94\% da população do Estado de Alagoas, sendo considerada a cidade mais populosa.

A população do estudo foi composta pelos casos de SC, residentes em Maceió, que foram notificados no período de 2009 a 2018. A pesquisa teve como fonte de dados o Sistema de Informação em Saúde- SIS. Foram colhidos dados secundários das seguintes fontes: Sistema de Informação de Agravos de Notificação - SINAN: casos de SC notificados no período do estudo; Sistema de Informações sobre Nascidos Vivos - SINASC: nascimentos no período do estudo; Sistema de Informações sobre Mortalidade - SIM: óbitos no período do estudo.

Os dados foram extraídos dos sistemas supracitados junto à Secretaria de Estado de Saúde de Alagoas, pertencente a Superintendência de Vigilância em Saúde, setor de Gerência de Vigilância e Controle de Doenças Transmissíveis do Programa Estadual de Infecções Sexualmente Transmissíveis /AIDS e Hepatites Virais e Secretaria Municipal de Saúde de Maceió, setor de Vigilância Epidemiológica.

As etapas de coleta de dados, compreenderam a seleção dos casos de SC notificados no SINAN; a seleção dos óbitos fetais e não fetais registrados no SIM e classificados com causa básica e/ou causas associadas cujos códigos variaram de A50.0 a A50.9 da CID-10 - Classificação Estatística Internacional de Doenças e Problemas Relacionados à Saúde; e a seleção dos nascimentos no período de estudo, através do SINASC. 
Para evitar duplicação, os casos e óbitos identificados em cada sistema foram compatibilizados em relação às seguintes variáveis-chave: data de nascimento do RN, bairro, idade da mãe e sexo do RN.

Para cada ano do estudo, foi calculado as seguintes taxas: incidência, mortalidade infantil, mortalidade proporcional, crescimento e letalidade. Os dados para calcular as referidas taxas foram extraídos através do SINASC (dados de nascidos vivos), SIM (óbitos por SC e óbitos infantis) e SINAN (casos de SC). Todas as taxas foram calculadas por ano, para o período de 2009 a 2018.

Para caracterizar a assistência prestada aos casos de SC, foi empregado o SINAN a fim de calcular a frequência absoluta e relativa de variáveis dos seguintes blocos que constam na Ficha Individual de Investigação: antecedentes epidemiológicos da gestante/mãe; dados de laboratório, dados da gestante/mãe e tratamento da gestante/mãe. Estes dados foram expressos em tabelas de frequência e gráficos.

Para avaliar a associação entre as características demográficas, nível educacional, acesso ao pré-natal de mães residentes em Maceió, incidência de sífilis e evolução da SC, foram realizadas as seguintes análises: teste de qui-quadrado para variáveis categóricas, ANOVA para comprar variáveis contínuas e categóricas, bem como foi realizada Regressão Linear para avaliar a evolução da Incidência e Mortalidade da SC durante o período estudado. A significância adotada foi de p<0,05.

A pesquisa foi submetida ao Comitê de Ética em Pesquisa com Seres Humanos (CEP) através da Plataforma Brasil, e foi aprovada sob parecer $\mathrm{n}^{\circ} 3.138 .921$, em 09 de fevereiro de 2019, obedecendo às normas que regem a Resolução $\mathrm{n}^{\circ} 466 / 12$ do Ministério da Saúde e sua complementar Resolução 510/2016.

\section{Resultados}

No período de 2009 a 2018, foram notificados no SINAN 1.876 casos de sífilis congênita no município de Maceió, Alagoas. Como demostrado no gráfico 1, a frequência da SC apresentou tendência de aumento nos últimos 10 anos, sendo registrado a menor taxa de incidência em 2009 (5,7 por mil nascidos vivos) e maior taxa em 2014 (15,3 por mil nascidos vivos), indicando um aumento de 168,4\%. No ano de 2018 a taxa de incidência atingiu valores representativos (15,2 por mil nascidos vivos).

Gráfico 1 - Taxa de incidência de sífilis congênita (por 1.000 nascidos vivos), segundo ano de notificação, Maceió, 2009 a 2018.

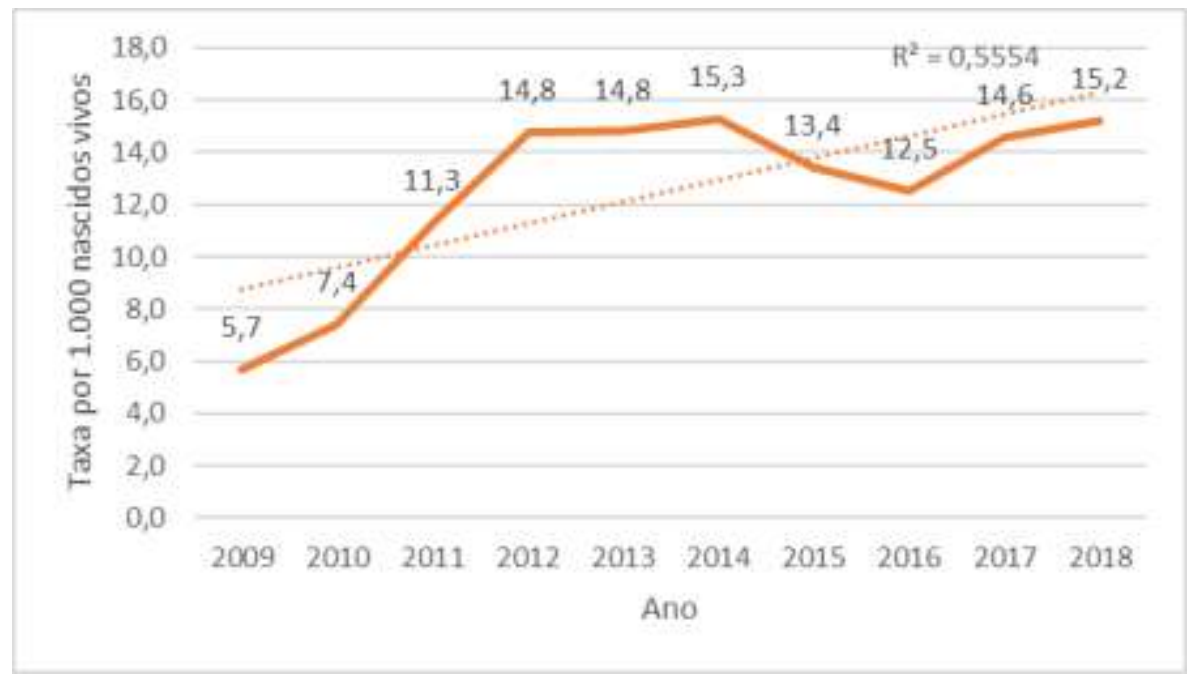

Fonte: SINAN/SESAU/SMS, Maceió (2019). 
Entre os períodos de 2009 a 2018, foram registrados 32 óbitos por sífilis congênita, o que correspondeu a uma letalidade de 1,7 óbitos/100 casos de SC. Em 2011 a taxa de letalidade atingiu (4,0 óbitos/100 casos de SC) e em 2012 (3,2 óbitos/100 casos de SC), em ambos os anos, registrou-se os maiores números de óbitos (7 casos) e as maiores taxas de letalidade.

A taxa de mortalidade infantil pela doença variou de 0 em 2009/2016 para 0,47 por mil nascidos vivos em 2012, mantendo um padrão regular entre os anos avaliados. Verificou-se que a proporção de óbitos por SC em menores de um ano também teve variação, atingindo o maior valor de 3,05\% em 2012 e menor valor em 2009 e 2016, por não haver registros de óbitos nesses dois anos.

A taxa de crescimento da doença sofreu flutuação ao longo do período do estudo, iniciando com (9,9\%) em 2009, atingindo seu maior pico em 2011 (54,9\%). Entre 2012 - 2016 ocorreu um declínio das taxas, chegando atingir valores (15,3\%), porém, em 2017 a taxa voltou a se elevar (19,8\%), diminuindo seus valores em 2018 (8,0\%). A taxa total de crescimento entre 2009-2018, foi de $157 \%$.

Quanto à proporção dos casos notificados de $\mathrm{SC}$ de acordo com a distribuição nos distritos sanitários do município de Maceió, os dados indicaram que todas as regiões distritais apresentaram crescimento nas taxas de incidência. O maior número de casos da doença foi concentrado no VII distrito 395 (25,7\%), seguido pelo II distrito 326 (21,2\%). Os bairros mais acometidos pelo agravo foram: Tabuleiro dos Martins, Cidade Universitária e Clima Bom (VII distrito); Vergel, Centro, Trapiche e Levada (II distrito).

A análise do perfil sociodemográfico das mulheres que tiveram filhos diagnosticados com SC revelou que estas são em sua maioria mulheres jovens, com idade média de 24,03 anos, variando entre 10 e 45 anos, sendo $25 \%$ da população composta por adolescentes. Acerca da raça/cor da pele auto declarada pelas mães, 1648 (94,2\%) declararam-se não brancas e $101(5,8 \%)$ brancas.

No que diz respeito à escolaridade, a maior parcela dessas mulheres $648(42,8 \%)$ declararam ter até 8 anos de estudo. A frequência da escolaridade sofreu mudança ao longo do período; houve redução de mulheres analfabetas e com idade de até 4 anos de estudo.

Os dados sociodemográficos das crianças com sífilis congênita, demostraram que a idade no momento da notificação variou entre 0 a 366 dias, com média de 2,87 e desvio padrão de 20,8, tendo p-valor 0,334. A variável sexo apresentou valores aproximados quando as duas classificações (masculino e feminino) e quanto a raça predominou crianças não brancas 1444 (95\%), seguida das brancas com 76 (5\%), conforme descrito na Tabela 1. 
Tabela 1 - Distribuição dos casos de sífilis congênita, segundo características sociodemográficas maternas e da criança, Maceió, 2009 a 2018.

\begin{tabular}{|c|c|c|c|c|c|c|c|c|c|c|}
\hline \multirow{2}{*}{ Características } & \multicolumn{2}{|c|}{ Vivo } & \multicolumn{2}{|c|}{ Óbito Sífilis } & \multicolumn{2}{|c|}{ Aborto } & \multicolumn{2}{|c|}{ Natimorto } & \multirow[t]{2}{*}{ Total } & \multirow[t]{2}{*}{ p-valor* } \\
\hline & $\mathrm{n}$ & $\%$ & $\mathrm{n}$ & $\%$ & $\mathrm{~N}$ & $\%$ & $\mathrm{n}$ & $\%$ & & \\
\hline \multicolumn{11}{|l|}{ Maternas } \\
\hline \multicolumn{11}{|l|}{ Raça } \\
\hline Branca & 86 & 5,7 & 2 & 6,5 & 4 & 4,8 & 9 & 7,6 & 101 & \multirow{5}{*}{$0,012 *$} \\
\hline Preta & 37 & 2,4 & 1 & 3,2 & 3 & 3,6 & 5 & 4,2 & 46 & \\
\hline Amarela & 2 & 0,1 & 0 & 0,0 & 0 & 0,0 & 0 & 0,0 & 2 & \\
\hline Parda & 1390 & 91,7 & 27 & 87,1 & 77 & 91,7 & 104 & 88,1 & 1598 & \\
\hline Indígena & 1 & 0,1 & 1 & 3,2 & 0 & 0,0 & 0 & 0,0 & 2 & \\
\hline \multicolumn{11}{|l|}{ Escolaridade } \\
\hline Analfabeto & 49 & 3,7 & 0 & 0,0 & 4 & 6,7 & 4 & 4,3 & 57 & \multirow{9}{*}{0,718} \\
\hline $1^{\mathrm{a}}$ a $4^{\mathrm{a}}$ série incompleta & 160 & 12,1 & 3 & 10,7 & 9 & 15,0 & 14 & 15,1 & 186 & \\
\hline $4^{\mathrm{a}}$ série completa & 131 & 9,9 & 6 & 21,4 & 3 & 5,0 & 6 & 6,5 & 146 & \\
\hline $5^{\mathrm{a}}$ a $8^{\mathrm{a}}$ incompleta & 577 & 43,5 & 11 & 39,3 & 19 & 31,7 & 41 & 44,1 & 648 & \\
\hline $\begin{array}{l}\text { Ensino fundamental } \\
\text { completo }\end{array}$ & 106 & 8,0 & 2 & 7,1 & 5 & 8,3 & 5 & 5,4 & 118 & \\
\hline Ensino médio incompleto & 108 & 8,2 & 1 & 3,6 & 9 & 15,0 & 9 & 9,7 & 127 & \\
\hline Ensino médio completo & 172 & 13,0 & 4 & 14,3 & 10 & 16,7 & 13 & 14,0 & 199 & \\
\hline Superior incompleto & 13 & 1,0 & 1 & 3,6 & 1 & 1,7 & 1 & 1,1 & 16 & \\
\hline Superior completo & 9 & 0,7 & 0 & 0,0 & 0 & 0,0 & 0 & 0,0 & 9 & \\
\hline \multicolumn{11}{|l|}{ Criança } \\
\hline \multicolumn{11}{|l|}{ Sexo } \\
\hline Feminino & 779 & 50,0 & 13 & 58,3 & 7 & 33,3 & 57 & 52,8 & 856 & \multirow{2}{*}{0,420} \\
\hline Masculino & 780 & 50,0 & 19 & 41,7 & 14 & 66,7 & 51 & 47,2 & 864 & \\
\hline \multicolumn{11}{|l|}{ Raça } \\
\hline Branca & 69 & 4,9 & 1 & 4,0 & 0 & 0 & 6 & 9,7 & 76 & \multirow{5}{*}{$0,000 *$} \\
\hline Preta & 25 & 1,8 & 0 & 0 & 2 & 12,5 & 1 & 1,6 & 28 & \\
\hline Amarela & 2 & 0,1 & 0 & 0 & 0 & 0 & 0 & 0 & 2 & \\
\hline Parda & 1320 & 93,2 & 23 & 92,0 & 14 & 87,5 & 55 & 88,7 & 1412 & \\
\hline Indígena & 1 & 0,1 & 1 & 4,0 & 0 & 0 & 0 & 0 & 2 & \\
\hline
\end{tabular}

Notas: $* \mathrm{p}<0,05$.

Fonte: SINAN/SESAU, Maceió (2019).

A análise dos casos de SC segundo a assistência ofertada às mães durante o período gravídico, mostrou que 1305 $(80,2 \%)$ mães realizaram pelo menos uma consulta de pré-natal, porém mais da metade dessas mulheres, 941 (53,2\%) tiveram seu diagnóstico somente durante o momento do parto/curetagem, não sendo garantido um diagnóstico em tempo oportuno.

Quanto à distribuição das mães com testagem para sífilis durante o parto/curetagem, $1796(99,4 \%)$ casos realizaram testes não treponêmicos, sendo reagente em 1783 casos e com teste treponêmico em 580 (34,6\%) casos.

Observa-se na Tabela 2, que a maioria das gestantes e parcerias sexuais não fizeram tratamento para sífilis, impedindo a quebra da cadeia de transmissão vertical. A porcentagem de gestantes com tratamento adequado foi de 97 (5,6\%) casos, sendo que em 2009, 2010, 2011 e 2012 não foram registrados nenhum caso com essa classificação.

Tabela 2 - Distribuição dos casos de sífilis congênita, segundo as características maternas da gestação, Maceió, 2009 a 2018. 
Research, Society and Development, v. 10, n. 5, e37110515042, 2021

(CC BY 4.0) | ISSN 2525-3409 | DOI: http://dx.doi.org/10.33448/rsd-v10i5.15042

\begin{tabular}{|c|c|c|c|c|c|c|c|c|c|c|}
\hline \multirow{2}{*}{ Características } & \multicolumn{2}{|c|}{ Vivo } & \multicolumn{2}{|c|}{ Óbito Sífilis } & \multicolumn{2}{|c|}{ Aborto } & \multicolumn{2}{|c|}{ Natimorto } & \multirow[t]{2}{*}{ Total } & \multirow[t]{2}{*}{ p-valor* } \\
\hline & $\mathrm{n}$ & $\%$ & $\mathrm{n}$ & $\%$ & $\mathrm{~N}$ & $\%$ & $\mathrm{n}$ & $\%$ & & \\
\hline \multicolumn{11}{|l|}{ Pré-natal } \\
\hline Sim & 1219 & 83,8 & 17 & 68,0 & 13 & 21,7 & 56 & 63,6 & 1305 & \multirow{2}{*}{$0,000^{*}$} \\
\hline Não & 235 & 16,2 & 8 & 32,0 & 47 & 78,3 & 32 & 36,4 & 322 & \\
\hline \multicolumn{11}{|c|}{ Diagnóstico de sífilis } \\
\hline Pré-natal & 548 & 35,9 & 8 & 25,0 & 1 & 1,1 & 13 & 10,9 & 570 & \multirow{4}{*}{$0,000 *$} \\
\hline Parto/curetagem & 744 & 48,8 & 23 & 71,9 & 80 & 88,9 & 94 & 79,0 & 941 & \\
\hline Após o parto & 230 & 15,1 & 1 & 3,1 & 9 & 10,0 & 12 & 10,1 & 252 & \\
\hline Não realizado & 4 & 0,3 & 0 & 0,0 & 0 & 0,0 & 0 & 0,0 & 4 & \\
\hline \multicolumn{11}{|c|}{ Teste não treponêmico ${ }^{1}$} \\
\hline Realizado & 1549 & 99,4 & 32 & 100,0 & 93 & 100,0 & 122 & 99,2 & 1796 & \multirow{2}{*}{0,919} \\
\hline Não realizado & 9 & 0,6 & 0 & 0,0 & 0 & 0,0 & 1 & 0,8 & 10 & \\
\hline \multicolumn{11}{|c|}{ Teste não treponêmico ${ }^{1}$} \\
\hline Reagente & 1537 & 98,7 & 32 & 100,0 & 92 & 98,9 & 122 & 99,2 & 1783 & \multirow{3}{*}{0,967} \\
\hline Não reagente & 12 & 0,8 & 0 & 0,0 & 1 & 1,1 & 0 & 0,0 & 13 & \\
\hline Não realizado & 9 & 0,6 & 0 & 0,0 & 0 & 0,0 & 1 & 0,8 & 10 & \\
\hline \multicolumn{11}{|c|}{ Teste treponêmico ${ }^{1}$} \\
\hline Reagente & 505 & 34,9 & 5 & 17,2 & 17 & 19,8 & 13 & 11,2 & 540 & \multirow{3}{*}{$0,000 *$} \\
\hline Não reagente & 34 & 2,4 & 0 & 0,0 & 4 & 4,7 & 2 & 1,7 & 40 & \\
\hline Não realizado & 906 & 62,7 & 24 & 82,8 & 65 & 75 & 101 & 87,1 & 1096 & \\
\hline \multicolumn{11}{|c|}{ Esquema de tratamento } \\
\hline Adequado & 95 & 6,4 & 1 & 3,2 & 1 & 1,2 & 0 & 0,0 & 97 & \multirow{3}{*}{$0,003 *$} \\
\hline Inadequado & 592 & 39,6 & 15 & 48,4 & 22 & 26,5 & 41 & 36,6 & 670 & \\
\hline Não realizado & 809 & 54,1 & 15 & 48,4 & 60 & 72,3 & 71 & 63,4 & 955 & \\
\hline \multicolumn{11}{|l|}{ Parceiro tratado } \\
\hline Sim & 200 & 15,1 & 2 & 8,0 & 4 & 6,1 & 10 & 10,8 & 216 & \multirow[t]{2}{*}{0,181} \\
\hline Não & 1125 & 84,9 & 23 & 92,0 & 62 & 93,9 & 83 & 89,2 & 1293 & \\
\hline
\end{tabular}

Notas: ${ }^{1}$ teste não treponêmico e treponêmico (ambos realizados no parto/curetagem), ${ }^{*} \mathrm{p}<0,05$.

Fonte: SINAN/SESAU, Maceió (2019).

No que diz respeito aos métodos de diagnóstico da criança, os dados afirmam que os casos notificados com SC realizaram teste não treponêmico de sangue periférico em 1384 (78,0\%) casos e os teste de seguimento se distribuíram da seguinte maneira: teste treponêmico, após 18 meses 18 (1,0\%) casos, teste de líquor 509 (29,3\%) casos, evidência treponêmica $377(23,1 \%)$ casos e raio $\mathrm{x}$ dos ossos longos $330(20,0 \%)$ casos, de acordo com os dados expostos na tabela abaixo. 
Tabela 3 - Distribuição dos casos de sífilis congênita, segundo os métodos de diagnóstico da criança, Maceió, 2009 a 2018.

\begin{tabular}{|c|c|c|c|c|c|c|c|c|c|c|}
\hline \multirow{2}{*}{ Características } & \multicolumn{2}{|c|}{ Vivo } & \multicolumn{2}{|c|}{ Óbito Sífilis } & \multicolumn{2}{|c|}{ Aborto } & \multicolumn{2}{|c|}{ Natimorto } & \multirow[t]{2}{*}{ Total } & \multirow[t]{2}{*}{ p-valor* } \\
\hline & $\mathrm{n}$ & $\%$ & $\mathrm{n}$ & $\%$ & $\mathrm{~N}$ & $\%$ & $\mathrm{n}$ & $\%$ & & \\
\hline \multicolumn{11}{|c|}{ Teste não treponêmico } \\
\hline Reagente & 970 & 63,2 & 7 & 21,9 & 1 & 1,2 & 0 & 0,0 & 978 & \multirow{3}{*}{$0,000 *$} \\
\hline Não reagente & 430 & 26,3 & 2 & 6,3 & 0 & 0,0 & 1 & 0,8 & 406 & \\
\hline Não realizado & 162 & 10,6 & 23 & 71,9 & 85 & 98,8 & 120 & 99,2 & 390 & \\
\hline \multicolumn{11}{|c|}{ Teste treponêmico $^{(1)}$} \\
\hline Reagente & 2 & 0,1 & 0 & 0,0 & 0 & 0,0 & 0 & 0,0 & 2 & \multirow{4}{*}{0,093} \\
\hline Não reagente & 16 & 1,1 & 0 & 0,0 & 0 & 0,0 & 0 & 0,0 & 16 & \\
\hline Não realizado & 243 & 16,3 & 3 & 9,4 & 5 & 5,6 & 9 & 7,4 & 260 & \\
\hline Não se aplica & 1229 & $82,5 \%$ & 29 & 90,6 & 84 & 94,4 & 113 & 92,6 & 1455 & \\
\hline \multicolumn{11}{|l|}{ Teste líquor } \\
\hline Reagente & 23 & 1,5 & 0 & 0,0 & 0 & 0,0 & 0 & 0,0 & 23 & \multirow{3}{*}{$0,000 *$} \\
\hline Não reagente & 485 & 32,4 & 1 & 3,1 & 0 & 0,0 & 0 & 0,0 & 486 & \\
\hline Não realizado & 989 & 66,1 & 31 & 96,9 & 85 & 100,0 & 122 & 100,0 & 1227 & \\
\hline \multicolumn{11}{|c|}{ Evidência treponêmica } \\
\hline Sim & 15 & 1,1 & 0 & 0,0 & 0 & 0,0 & 0 & 0,0 & 15 & \multirow{3}{*}{$0,000 *$} \\
\hline Não & 360 & 25,9 & 0 & 0,0 & 2 & 2,3 & 0 & 0,0 & 362 & \\
\hline Não realizado & 1016 & 73,0 & 30 & 100,0 & 84 & 97,7 & 121 & 100,0 & 1251 & \\
\hline \multicolumn{11}{|c|}{ Diagnóstico radiológico } \\
\hline Sim & 16 & 1,1 & 0 & 0,0 & 0 & 0,0 & 0 & 0,0 & 16 & \multirow{3}{*}{$0,000 *$} \\
\hline Não & 312 & 22,0 & 2 & 6,5 & 0 & 0,0 & 0 & 0,0 & 314 & \\
\hline Não realizado & 1088 & 76,8 & 29 & 93,5 & 85 & 100,0 & 120 & 100,0 & 1322 & \\
\hline
\end{tabular}

Notas: ${ }^{1}$ teste treponêmico (após 18 meses), $*$ p $<0,05$.

Fonte: SINAN/SESAU, Maceió (2019)

Considerando os aspectos clínicos relacionados à assistência neonatal das crianças notificadas com a doença pesquisada, a Tabela 4, traz a informação de que a maioria dos casos de SC foram diagnosticados como assintomáticos em $1262(82,0 \%)$ dos casos, e quando sintomático, o sinal de maior evidência foi a icterícia. Ainda no que diz respeito aos sinais e sintomas, os casos registrados como outros trouxeram os seguintes dados: neurossífilis, lesões cutâneas, prematuridade, insuficiência respiratória, alterações cardiológicas, dentre outras.

O esquema de tratamento com penicilina G cristalina 100.000 a $150.00 \mathrm{UI} / \mathrm{kg} / \mathrm{dia}$, durante 10 dias, foi prescrito a 1292 $(88,0 \%)$ casos, variando apenas a dose, que foi de acordo com individualidade de cada caso. Na ficha de notificação, no campo outros, foram registrados outros tipos de antibioticoterapia que não são capazes de tratar a sífilis congênita.

Dos casos investigados, 1517 (83,7\%) foram classificados com diagnóstico final de sífilis recente, que é quando o diagnóstico ocorre nos dois primeiros anos de vida, sendo tardio em apenas $2(0,1 \%)$ casos. 
Tabela 4 - Distribuição dos casos de sífilis congênita, segundo dados clínicos e tratamento da criança, Maceió, 2009 a 2018.

\begin{tabular}{|c|c|c|c|c|c|c|c|c|c|c|}
\hline \multirow{2}{*}{ Características } & \multicolumn{2}{|c|}{ Vivo } & \multicolumn{2}{|c|}{ Óbito Sífilis } & \multicolumn{2}{|c|}{ Aborto } & \multicolumn{2}{|c|}{ Natimorto } & \multirow[t]{2}{*}{ Total } & \multirow[t]{2}{*}{ p-valor* } \\
\hline & $\mathrm{n}$ & $\%$ & $\mathrm{~N}$ & $\%$ & $\mathrm{n}$ & $\%$ & $\mathrm{~N}$ & $\%$ & & \\
\hline \multicolumn{11}{|l|}{ Diagnóstico clínico } \\
\hline Assintomático & 1243 & 82,8 & 8 & 38,1 & 6 & 100,0 & 5 & 83,3 & 1262 & \multirow{2}{*}{$0,000^{*}$} \\
\hline Sintomático & 259 & 17,2 & 13 & 61,9 & 4 & 0,0 & 1 & 16,7 & 277 & \\
\hline \multicolumn{11}{|l|}{ Sinais e sintomas ${ }^{1}$} \\
\hline Icterícia & 216 & 14,4 & 7 & 35,0 & 0 & 0,0 & 0 & 0,0 & 223 & 0,075 \\
\hline Anemia & 4 & 0,3 & 0 & 0,0 & 0 & 0,0 & 0 & 0,0 & 4 & 0,999 \\
\hline Rinite & 15 & 1,0 & 2 & 10,0 & 0 & 0,0 & 0 & 0,0 & 17 & $0,000^{*}$ \\
\hline Esplenomegalia & 16 & 1,1 & 2 & 10,0 & 0 & 0,0 & 0 & 0,0 & 18 & $0,000^{*}$ \\
\hline Hepatomegalia & 32 & 2,1 & 4 & 20,0 & 0 & 0,0 & 0 & 0,0 & 36 & $0,000^{*}$ \\
\hline Osteocondrite & 5 & 0,3 & 0 & 0,0 & 0 & 0,0 & 0 & 0,0 & 5 & 0,998 \\
\hline Lesões cutâneas & 12 & 0,8 & 0 & 0,0 & 0 & 0,0 & 0 & 0,0 & 12 & 1,000 \\
\hline Pseudopbaralisia & 1 & 0,1 & 0 & 0,0 & 0 & 0,0 & 0 & 0,0 & 1 & 0,989 \\
\hline Outros & 53 & 3,6 & 12 & 57,1 & 0 & 0,0 & 0 & 0,0 & 65 & $0,000 *$ \\
\hline \multicolumn{11}{|l|}{ Esquema de tratamento } \\
\hline Penicilina G cristalina & 1289 & 88,8 & 2 & 18,2 & 0 & 33,3 & 0 & 0,0 & 1292 & \multirow{4}{*}{$0,000^{*}$} \\
\hline Penicilina G procaína & 20 & 1,4 & 1 & 9,1 & 0 & 0,0 & 0 & 0,0 & 21 & \\
\hline Penicilina $\mathrm{G}$ benzatina & 20 & 1,4 & 0 & 0,0 & 0 & 66,7 & 0 & 100,0 & 24 & \\
\hline Outros & 122 & 8,4 & 8 & 72,7 & 0 & 0,0 & 0 & 0,0 & 130 & \\
\hline \multicolumn{11}{|l|}{ Diagnóstico final } \\
\hline Recente & 1486 & 95,0 & 31 & 96,9 & 0 & 0,0 & 0 & 0,0 & 1517 & \multirow{5}{*}{$0,000^{*}$} \\
\hline Tardia & 2 & 0,1 & 0 & 0,0 & 0 & 0,0 & 0 & 0,0 & 2 & \\
\hline Aborto & 0 & 0,0 & 0 & 0,0 & 91 & 98,9 & 0 & 0,0 & 91 & \\
\hline Natimorto & 0 & 0,0 & 0 & 0,0 & 0 & 0,0 & 123 & 100,0 & 123 & \\
\hline Descartado & 76 & 4,9 & 1 & 3,1 & 1 & 1,1 & 0 & 0,0 & 78 & \\
\hline
\end{tabular}

Notas: ${ }^{1}$ Dados referentes aos casos positivos para os respectivos sinais e sintomas, ${ }^{2}$ Descartado - investigação incompleta impossibilitando o diagnóstico final do caso, ${ }^{*} \mathrm{p}<0,05$.

Fonte: SINAN/SESAU, Maceió (2019).

A evolução do caso teve como desfecho o RN vivo em 1488 (82,2\%) casos, 31 (1,8\%) óbitos por sífilis congênita, 91 $(5,2 \%)$ casos de aborto e $123(7,1 \%)$ natimorto, os demais casos foram registrados como sendo óbito por outras causas e ignorado.

\section{Discussão}

O crescente aumento da taxa de incidência de sífilis congênita no mundo destaca a magnitude do problema, de forma que, muitos estudos buscam identificar o comportamento da doença ao longo dos anos e as estratégias de prevenção, como resposta para o seu controle. Os resultados do presente estudo apontaram a relevância do problema baseando-se no aumento na taxa de incidência de sífilis congênita no município de Maceió/Alagoas, com taxas superiores ao proposto para erradicação da doença.

Dentre o período de 2009 a 2018, o município atingiu a maior taxa de incidência por SC em 2014, com 15,3 casos por 1.000 nascidos vivos. Dado aproximado foi observado no município de Catanduva-SP, com uma incidência de 14,5/1.000 nascidos vivos no período de 2014 (Tannous et al., 2017). Comparando esses valores com os dados publicados no Boletim Epidemiológico de Sífilis, é possível verificar que houve, nos últimos dez anos, um aumento na taxa de incidência de SC em 
todo o território brasileiro (Brasil, 2018a).

Considerando que desde o ano de 2014, vem sendo registrado mundialmente um desabastecimento da penicilina, este fato pode estar relacionado ao aumento dos índices de SC nesse período (Fernandes et al., 2017; Ferreira, Barbosa \& Maleck, 2018; Ferreira et al., 2018).

É possível justificar esse aumento na incidência da doença de acordo com as seguintes hipóteses: 1) melhoria na notificação e investigação dos casos, 2) ampliação dos critérios de definição de caso de sífilis congênita ocorrida no ano de 2004, 3) necessidade da realização do teste não treponêmico (VDRL) no momento do parto como requisito para o recebimento do pagamento do procedimento pelos hospitais conveniados ao SUS, 4) deficiência na qualidade da assistência de pré-natal, 5) dificuldade de acesso ao serviço de saúde e desestruturação do sistema, 6) manutenção da transmissão vertical por diminuição do uso dos métodos contraceptivos, especialmente do preservativo (camisinha) (França et al., 2015; Nonato et al., 2015; Feitosa, Rocha \& Costa, 2016).

Nesse contexto epidemiológico de aumento de casos de sífilis, no que refere à mortalidade por esta causa, no Brasil, a taxa de mortalidade infantil por sífilis congênita oscilou de 2,3/100 mil nascidos vivos em 2007 para 7,2/100 mil nascidos vivos em 2017 (Brasil. 2018a). No presente estudo, a taxa de mortalidade infantil foi menor do que os níveis nacionais, variando entre 0 a 0,4 mortes por 1.000 nascidos vivos. Essa realidade também foi observada em outros estudos que evidenciam a SC como importante fator para a mortalidade infantil (Domingues \& Leal, 2016; Guimarães et al., 2018).

Mesmo com taxas de mortalidade abaixo dos níveis nacionais, a manutenção dessa situação de saúde é considerada como um problema bastante relevante, por se tratar de mortes evitáveis, sinalizando a necessidade de uma maior atenção voltada para esta causa, em busca de uma resposta eficiente do setor saúde para controle do agravo (Reis et al., 2018).

Foi observado nesse estudo, que fatores sociodemográficos maternos podem sinalizar o risco e a vulnerabilidade em adquirir sífilis congênita, como idade materna entre 20-29 anos, baixa escolaridade e cor de pele parda. Convém ressaltar que muitos estudos documentam sobre essa associação mencionada, semelhança encontrada nos estudos (Alves et al., 2016; Moreira et al., 2017; Almeida \& Pinto, 2018; Cardoso et al., 2018; Gonçalves Trevisan et al., 2018) que reforçam esses achados.

O perfil epidemiológico das crianças com sífilis congênita desse estudo, apresentou valores aproximados em relação ao sexo e obteve predomínio da raça parda. Estudos nacionais reforçam esse perfil, mostrando valores proporcionalmente semelhantes (Magalhães et al., 2013; Carlos \& Brito, 2014; Moreira et al., 2017; Sandes et al., 2019; Silva, Sousa \& Sakae, 2017). A faixa etária encontrada corrobora outros autores (Guimarães et al., 2018; Signor et al., 2018), acomete principalmente os menores de um ano.

Levando em consideração os dados de cobertura de pré-natal em Maceió, pode-se verificar que mais de $90 \%$ das gestantes foram atendidas por profissional da saúde com pelo menos uma consulta. Contudo, ainda se observa um elevado índice de transmissão vertical por sífilis, o que pode estar relacionado ao diagnóstico tardio de sífilis gestacional. De maneira semelhante, outros estudos corroboram com esses dados (Domingues \& Leal, 2016; Cardoso et al., 2018; Gonçalves Trevisan et al., 2018).

A qualidade na atenção ao pré-natal é uma estratégia importante na diminuição do número de casos de SC, podendo ter forte relação com os desfechos perinatais negativo, além de identificar e controlar outros fatores de riscos para a saúde materno-infantil (Carvalho \& Brito, 2014; Arruda \& Ramos, 2020). A privação da dessa assistência, pode aumentar em até cinco vezes a mortalidade perinatal (Magalhães et al, 2013).

O pré-natal representa um espaço de cuidado adequado para se trabalhar ações de promoção e prevenção da sífilis congênita, porém, ainda é possível verificar uma baixa adesão nas consultas e exames realizados pelas gestantes (Suto et al., 2016; Beck \& Souza, 2018). 
Mesmo com a ampliação da cobertura do diagnóstico sendo garantida pelo Ministério da Saúde, no presente estudo, houve um menor acesso ao diagnóstico de sífilis materna no pré-natal, porém com diagnósticos significativos, mais de 90\%, no momento do parto. Assim, pode-se dizer que a assistência do durante o período gestacional está aquém do preconizado, revelando uma assistência precária.

Este achado aproxima-se ao de uma pesquisa realizada entre 2011-2012, estudo nacional de base hospitalar, com 23.894 puérperas, com taxa de 60,3\% que não realizaram duas sorologias para a sífilis na gestação (Domingues \& Leal, 2016). Entretanto, resultados diferentes foram observados em outras realidades brasileiras (Boni \& Pagliari, 2016; Moreira et al., 2017; Cardoso et al., 2018).

No que diz respeito ao tratamento da gestante e do seu parceiro sexual, os resultados desse estudo apontam para um grande problema de saúde. Tendo em vista que a maioria das mães realizaram acompanhamento de pré-natal, e apenas 5,6\% delas, tiveram tratamento adequado e 14,3\% dos parceiros sexuais foram tratados. Dado confirmado por outras pesquisas (Magalhães et al., 2013; Carvalho \& Brito, 2014; Moreira et al., 2017; Cardoso et al., 2018; Jesus \& Mafra, 2019).

Dessa forma, observa-se que a sífilis congênita tem grande repercussão no cenário de saúde pública em nível local e nacional, pois nos mostra indicadores importantes a respeito da qualidade do pré-natal. Um pré-natal de alta qualidade possibilita uma abordagem eficaz, da gestante infectada com tratamento oportuno desta, e dos seus parceiros sexuais (Motta et al., 2018). Um fator de preocupação está nessa assistência prestada durante o período gestacional, visto que é possível detectar a doença, dar seguimento adequado em tempo oportuno, diminuindo assim a transmissão vertical por esta causa.

Quanto aos dados laboratoriais da criança, observou-se que os exames de diagnóstico, VDRL de sangue periférico, foram realizados na maioria; porém, os exames de seguimento não foram realizados ou ignorados, semelhança encontrada em outros estudos (Cavalcante et al., 2017; Moreira et al., 2017).

Estes exames são de suma importância para o acompanhamento da criança frente à infecção. Pesquisa conduzida em Palmas (Carvalho \& Brito, 2014), no período de 2007-2014, também mostrou essa falha no diagnóstico dos casos com sífilis congênita, fato também encontrado em Porto Velho (Moreira et al., 2017), pois 45,2\% dos casos, não realizaram nenhum desses exames.

No município de Maceió, mesmo com registro de tratamento adequado dos RN diagnosticados com sífilis congênita, não há referência, nem garantia de seguimento clínico e sorológico na rede, sendo uma grande limitação para controle da doença. No estudo realizado no Distrito Federal (Magalhães et al., 2013) em maternidades públicas, também observou a ocorrência da falha no seguimento do RN após a alta da maternidade, não existe anotações a respeito de encaminhamentos à rede de atenção básica para o seguimento ambulatorial.

O diagnóstico final dos casos desse estudo foi, predominantemente, sífilis congênita recente. Sendo corroborado com outros estudos (Guimarães et al., 2018; Signor et al., 2018).

Quanto à presença de sinais e sintomas, os dados desta pesquisa confirmam que a maior parcela das crianças foi diagnosticada sem sintomas, dado equivalente ao encontrado em estudo realizado em Fortaleza - CE com valores de 114 (67,5\%) casos (Cardoso et al., 2018).

Nesse estudo, o esquema de tratamento seguiu o preconizado pelos órgãos de saúde, a maior parcela das crianças notificadas com sífilis congênita foi tratada com Penicilina G cristalina. Dado semelhante ao encontrado em outro estudo (Moreira et al., 2017).

A sífilis congênita é um marcador da qualidade da assistência prestada à saúde materno-infantil, diante das estratégias para seu controle, como o diagnóstico de baixo custo e o tratamento acessível por meio de medicação eficiente. Portanto, o aumento das taxas de incidência é indicador de falhas na atenção ao pré-natal. As informações apresentadas nesse estudo servirão como informações para auxiliar no planejamento e monitoramento mais efetivos da sífilis congênita, a fim de que 
ocorra uma mudança no quadro epidemiológico.

Analisando a situação do município frente aos casos de sífilis congênita, observou-se com esse estudo uma limitação quanto a subnotificação dos dados. No banco de dados do estudo houve variáveis relevantes que não foram preenchidas ou foram de forma incorreta. Esses dados de subnotificação aliados à baixa qualidade dos registros são considerados como entraves para alcançar a erradicação da doença na população (Lafetá et al, 2016).

\section{Conclusão}

O estudo constatou que os dados apresentados são relevantes sobre a atual situação de saúde do município de Maceió frente aos casos de sífilis congênita. A partir dos resultados, pode-se afirmar que a incidência de sífilis congênita apresenta taxas crescentes no período de 10 anos e taxa de mortalidade constante.

No tocante à evolução dos casos, os dados revelam que a assistência de pré-natal impacta diretamente nas complicações da doença, com taxas de letalidade significativas. Tal fato é considerado um problema de saúde pública, devendo ser investido estratégias mais eficazes voltadas para controle da doença, visto que é uma infecção passível de prevenção durante o pré-natal.

\section{Agradecimentos}

Agradecemos à Fundação de Amparo à Pesquisa do Estado de Alagoas por contribuir como fonte de financiamento para o desenvolvimento da pesquisa e à Secretária do Estado de Saúde de Alagoas pelo fornecimento dos dados e disponibilidade em elucidar os questionamentos da referida pesquisa.

\section{Referências}

Almeida, F. C. M., \& Pinto, F. J. M. Transmissão vertical da sífilis: análise dos fatores intervenientes na morbimortalidade. EdUECE, 2018. Livro eletrônico. 98p.

Alves, W. A., Cavalcanti, G. R., Nunes, F. A., Teodoro, W. R., Carvalho, L. M., \& Domingos, R. S. Sífilis Congênita: Epidemiologia dos casos notificados em Alagoas, Brasil, 2007 a 2011. Revist. Port.: Saúde e Sociedade. 1(1):27-41, 2016.

Arruda, L. R., \& Ramos, A. R. S. Importância do diagnóstico laboratorial para a sífilis congênita no pré-natal. J Manag Prim Health Care, $2020,12:$ : 12.

Barbosa, D. R. M, Almeida, M. G., Silva, A. O., Araújo, A. A., \& Santos, A. G. Perfil epidemiológico dos casos de sífilis gestacional. Rev Enferm UFPE. 2017,11(5):1867-1874.

Beck, E. Q., \& Souza, M. H. T. Práticas de enfermagem acerca do controle da sífilis congênita. Rev. Pesqui. Cuid. Fundam. 2018, 10(3):19-24.

Brasil. Ministério da Saúde. Guia de Vigilância em Saúde: volume único (recurso eletrônico). 2017. http://portalarquivos.saude.gov.br/images/pdf/2017/outubro/06/Volume-Unico-2017.pdf.

Brasil. Secretaria de Vigilância em Saúde. Ministério da Saúde. Boletim Epidemiológico. 2018. http://www.aids.gov.br/pt-br/pub/2018/boletimepidemiologico-de-sifilis-2018.

Brasil. Ministério da Saúde. Protocolo clínico e diretrizes terapêuticas para Prevenção da Transmissão Vertical de HIV, Sífilis e Hepatites Virais. 2018. http://www.aids.gov.br/pt-br/pub/2015/protocolo-clinico-e-diretrizes-terapeuticas-para-prevencao-da-transmissao-vertical-de-hiv.

Brasil. Ministério da Saúde. Protocolo Clínico e Diretrizes Terapêuticas para Prevenção da Transmissão Vertical de HIV, Sífilis e Hepatites Virais. 2019. http://www.aids.gov.br/pt-br/pub/2015/protocolo-clinico-e-diretrizes-terapeuticas-para-prevencao-da-transmissao-vertical-de-hiv.

Brasil. Ministério da Saúde. Departamento de Saúde da Família. Informação e Gestão da Atenção Básica. 2019. https://egestorab.saude.gov.br/paginas/acessoPublico/relatorios/relHistoricoCoberturaAB.xhtml.

Boni, S. M., \& Pagliari, P. B. Incidência de sífilis congênita e sua prevalência em gestantes em um município do Noroeste do Paraná. Revista Saúde e Pesquisa. 2016, 9(3):517-524.

Cardoso, A. R. P., Araújo, M. A. L., Cavalcante, M. S., Frota, M. A., \& Melo, S. P. Análise dos casos de sífilis gestacional e congênita nos anos de 2008 a 2010 em Fortaleza, Ceará, Brasil. Ciência \& Saúde Coletiva, 2018, 23(2):563-574, 2018.

Carvalho, I. S., \& Brito, R. S. Sífilis congênita no Rio Grande do Norte: estudo descritivo do período 2007-2010. Epidemiol. Serv. Saúde. 2014,23(2):287-94. 
Cavalcante, P. A. M., Pereira, R. B. L., \& Castro, J. G. D. Sífilis gestacional e congênita em Palmas. Epidemiol. Serv. Saúde. 2017, $26(2): 255-264$.

Coelho, J. M. R. et al. Sífilis: um panorama epidemiológico do Brasil e do município de Volta Redonda/RJ. Braz. J. Hea. Rev. 2018, 1(1): 128-147.

Costa, C. C., Freitas, L. V., Sousa, D. M. N., Oliveira, L. L., Chagas, A. G. M. A., Lopes, M. V. O., Damasceno, A. K. C. Sífilis congênita no Ceará: análise epidemiológica de uma década. Rev. Esc. Enferm. USP. 2013,47(1):152-159.

Cerqueira, L. R. P., Monteiro, D. L. M., Taquette, S. R., Rodrigues, N. C. P., Trajano, A. J. B., Souza, F. M., Araújo, B. M. The magnitude of syphilis: from prevalence to vertical transmission. Rev. Inst. Med. Trop. 2017,59.

Domingues, R. M. S. M., Leal, M. C. Incidência de sífilis congênita e fatores associados à transmissão vertical da sífilis: dados do Estudo Nascer no Brasil. Cad. Saúde Pública. 2016,32(6).

Feitosa, J. A. S., Rocha, C. H. R., Costa, F. S. Sífilis congênita. Rev. Med. Saúde Brasília. 2016 , 5(2):286-297. https://portalrevistas.ucb.br/index.php/rmsbr/article/view/6749/4573.

Fernandes, A. A. S., Regina, A. L. A., Ladvocat, A. C. C. P., Felix, J. C. M., Ishii, J. S. C., Chellini, P. R. Prevalência de resultados positivos de VDRL (Venereal Disease Research Laboratory) e análise das variáveis epidemiológicas em pacientes atendidos no serviço de saúde pública. HU Revista. 2017,43(3):383-390.

Ferreira, A. G., Gomes, L. A., Medeiros, R. C. T., Leonidas, R. A. M., Medeiros, J. P. Perfil dos Casos de Sífilis Congênita no Município de Natal / RN no Período de 2007 a 2015. Revista Saúde em Foco. 2018,5(1):3-27.

Ferreira, A. G., Barbosa, V. F. J., Maleck, M. Comparação entre a incidência, nacional, estadual e do município de Vassouras, de sífilis congênita, nos últimos quinze anos. Revista de Saúde. 2018,9(1):14-17.

França, I. S. X., Batista, J. D. L., Coura, A. S., Oliveira, C. F., Araújo, A. K. F., Sousa, F. S. Fatores associados à notificação da sífilis congênita: um indicador de qualidade da assistência pré-natal. Rev Rene. 2015,16(3):374-81.

Gonçalves Trevisan, M., et al. Prevalência da sífilis gestacional e congênita no município de Francisco Beltrão. Rev Espaço para a Saúde. 2018,19(2):84-96. https://doi.org/10.22421/15177130-2018v19n2p84.

Guimarães, T. A., Alencar, L. C. R., Fonseca, L. M. B., Gonçalves, M. M. C., Silva, M. P. Sífilis em gestantes e sífilis congênita no Maranhão. Arq. Ciênc. Saúde. 2018,25(2):24-30.

Jesus, T. B. S., Mafra, A. L. S. Sífilis em gestante e congênita: casos notificados de um município do Noroeste Paulista. Revista Nursing. 2019, 22(250):27662771 .

Lafetá, K. R. G., Marteline, H. J., Silveira, M. F., Paranaiba, L. M. R. Sífilis materna e congênita, subnotificação e difícil controle. Rev. Bras. Epidemiol. $2016,19(1)$

Magalhães, D. M. S., Kawaguehi, I. A. L., Dias, A., Calderon, I. M. P. Sífilis materna e congênita: ainda um desafio. Cad. Saúde Pública. 2013,29(6):11091120 .

Mahmud, I. C., et al. Sífilis adquirida: uma revisão epidemiológica dos casos em adultos e idosos no município de Porto Alegre/RS. Rev. Epidemiol. Controle Infecç. Santa Cruz do Sul, 2019,9(2):177-184.

Moreira, K. F. A., Oliveira, D. M., Alencar, L. N., Cavalcante, D. F. B., Pinheiro, A. S., Orfão, N. H. Perfil dos casos notificados de sífilis congênita. Cogitare Enferm. 2017,22(2).

Motta, I. A., et al. Sífilis congênita: por que sua prevalência continua tão alta? Rev Med Minas Gerais. 2018, 28. http://rmmg.org/artigo/detalhes/2418.

Neves, K. do C., et al. O conhecimento do homem sobre a sífilis: Impacto nas ações preventivas e adesão ao tratamento. Saúde Coletiva (Barueri). 2019, 9(50):1789-1794. http://www.revistas.mpmcomunicacao.com.br/index.php/saudecoletiva/article/view/159

Nonato, S. M., Melo, A. P. S., Guimarães, M. D. C. Syphilis in pregnancy and factors associated with congenital syphilis in Belo Horizonte-MG, Brazil, 20102013. Epidemiol. Serv. Saúde [online]. 2015,24(4):681-694.

Reis, G. J., Barcelos, C., Pedrosa, M. M., Xavier, D. R. Diferenciais intraurbanos da sífilis congênita: análise preditiva por bairros do Município do Rio de Janeiro, Brasil. Cad. Saúde Pública. 2018,34(9).

Sandes, M. F., Mendonça, R. C., Alves, M. M. S., Santos, L. G., Lima, M. G., Farias, R. O., Torres, R. F. M. C., Bispo, A. J. B. Análise epidemiológica por sífilis congênita em menores de um ano no estado de Sergipe. Braz. J. Hea. Rev. 2019, 2(3):1609-1615. http://www.brjd.com.br/index.php/BJHR/article/view/1409.

Silva, H. C. G., Sousa, T. O., Sakae, T. M. Incidência de sífilis congênita no Estado de Santa Catarina no ano de 2012. Arq. Catarin Med. 2017, 46(2):15-25. http://www.acm.org.br/acm/seer/index.php/arquivos/article/view/265.

Signor, M., Spagnolo, L. M. L., Tomberg, J. O., Gabatto, M., Stofel, N. S. Spatial distribution and characterization of cases of congenital syphilis. Rev Enferm UFPE. 2018,12(2):398-406.

Sonda, E. C., Richeter, F. F., Boschetti, G., Casasola, M. P., Krumel, C. F., Machado, C. P. H. Sífilis Congênita: uma revisão da literatura. Rev. Epidemiol. Control. Infect. 2013,3(1):28-30.

Suto, C. S. S., Silva, D. L., Almeida, E. S., Costa, L. E. L., Evangelista, T. J. Assistência pré-natal a gestante com diagnóstico de sífilis. Rev Enferm Atenção Saúde. 2016,5(2):18-33. 
Research, Society and Development, v. 10, n. 5, e37110515042, 2021

(CC BY 4.0) | ISSN 2525-3409 | DOI: http://dx.doi.org/10.33448/rsd-v10i5.15042

Tannous, L. S. D., Pansiera, C. J., Ribeiro, M. P., Oliveira, M. S., Contiero, N. C. Comparação entre os índices de sífilis na gestação e sífilis congênita na região de Catanduva-SP. CuidArte. Enfermagem. 2017,11(2):187-192, 2017. http://www.webfipa.net/facfipa/ner/sumarios/cuidarte/2017v2/187.pdf. 